\title{
OPTIMIZATION OF ENERGY EXTRACTION USING DEFINITE GEOMETRY PRISMS IN AIRFLOW
}

\author{
I. Tipans, J. Viba, M. Irbe* and S. K. Vutukuru \\ Faculty of Mechanical Engineering, Transport and Aeronautics, \\ Riga Technical University, \\ 6B Kipsalas Str., LV-1048, Riga, LATVIA \\ *e-mail: martins.irbe@rtu.Iv
}

An approximate method for analysis and synthesis of moving rigid bodies (prisms) in the airflow without using numerical methods of space-time programming techniques is described by applying a fluid (air)-rigid solid body interaction concept for engineering applications through a straightforward mathematical model. The interaction of rigid body (prism) and air is encountered in different cases: moving body (prism) in the air; stationary bodies (prism) in the airflow; moving body (prism) in the airflow. The complicated task of rigid body (prism) and air interaction is simplified by using superposition principles, i.e., by taking into account the upstream and downstream rigid body (prism) and air interaction phenomenon, which has been found to be different under varying speeds. Numerical results obtained for various forms of prisms are shown for constant air-speed, where the steady state Reynoldsaveraged Navier-Stokes (RANS) equation is solved by using k- $\varepsilon$ realizable turbulence model. A detailed explanation to support the proposed approximate method is given by using numerical results obtained in ANSYS computations. All equations are formed based on laws of classical mechanics; the interaction of viscous forces is neglected in forming the mathematical model. Numerical results for different model prisms are compared and the theoretical results discussed in detail. The mathematical model in the present paper is applicable only to bodies that undergo a rectilinear translation motion. In the final part of the present paper, the proposed method is used in the synthesis and optimization task of energy extraction by considering the motion of a variable parameter prism in the airflow.

Keywords: Rigid body (prisms), superposition principle, space-time programming. 
The theme of the interaction between the solid (rigid) body and fluid still plays an important role in science, technology, production processes and in everyone's life.

In the present paper, three possible cases of fluid-rigid body interaction are studied: stationary rigid body in the air, non-stationary body in the air and non-stationary body in the airflow. Though good research pertaining to non-stationary rigid body-fluid interaction in fluid flows is available, no one can deny the fact that understanding the phenomena requires advanced programming skills (space-time techniques) along with in-depth knowledge of CFD and core engineering mathematics. General mesh deforming and re-meshing techniques are time consuming and strictly have geometry constrains and many times are computationally expensive. A better method compared to moving and deforming techniques with no restriction on closeness between objects and their complex motion is studied where continuity and momentum equations include motion effects [1]. Different types of mesh moving techniques for fluid-structure interaction were studied and compared. A new use of space-time discretization that could be extended for a full-time space Galerkin discretization was examined [2]. Fixed mesh technique by using arbitrary Lagrangian-Eulerian approach for flow problems in a moving domain was studied and for every time step results were displayed on a background mesh [3]-[7]. An algorithm for space-time finite element meshes was used to obtain numerical results for transient Navier-Strokes equations for an adaptive moving mesh in time [8]. New XFEM approach was proposed based on a fixed grid method, which did not take into account most of fluid unknown characteristics. This method is advantageous as simulations of deforming structures could be performed effectively [9]. Easily understandable and less complex method for space-time mesh that allows for refinement of the selected locations within the domain was also proposed [10]. Advantage of saving the computational time, accounting for flexibility of unstructured meshes and improving the scope of compressible fluid flow were mentioned in [11]. Solution for non-stationary fluid body problems by using a high-order finite element method based on space-time discontinuous Galerkin (DG) procedure was proposed and extended to explain the dynamics of parachute motion [12]. A simple method using the concept of zones, averting the space time programming techniques and extending the concept for energy extraction for simple form objects was performed in [13]. In the present study, without using any numerical space-time programming techniques and in a straightforward way using the averaged principle of superposition, fluid interaction with rigid body is explained. The entire paper specifically focuses solely on rigid body-fluid (air) interaction, does not consider flow reattachment or flow separation phenomenon and offers an alternative to a vortex induced vibration concept [14]-[16] and the same study is further extended for energy extraction by using variable parameters of definite geometry-prism. The main idea of the article is to propose a simplified approach to an interaction phenomenon and to offer an alternative to vortex induced vibration techniques through a mathematical model that does not consider the viscous effects of fluid (air) but the viscous effects are in a way indirectly resulting from the mathematical model by considering the output from steady state numerical simulations (RANS by $\mathrm{K}-\varepsilon$ turbulence model) in ANSYS. 


\section{MATHEMATICAL MODELS FOR FLUID (AIR) STRUCTURE INTERACTION}

Keeping in view the much-needed tasks of analysis, synthesis and optimization that are required in many engineering applications, the present paper offers an averaged mathematical model approximately assum- ing a laminar flow for the incompressible fluid. However, the mathematical model does not include the viscous nature of the fluid under consideration.

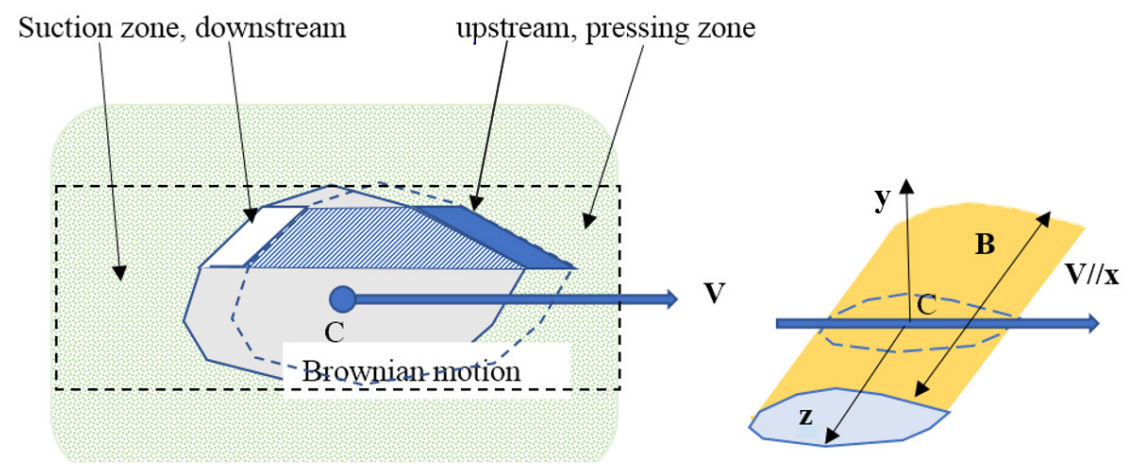

Fig. 1. External relative flow over a moving prism in very low speed airflow.

The space around the body under consideration when subjected to fluid is carefully studied; a straightforward mathematical model using classical laws of mechanics is explained based on a fluid structure inter-

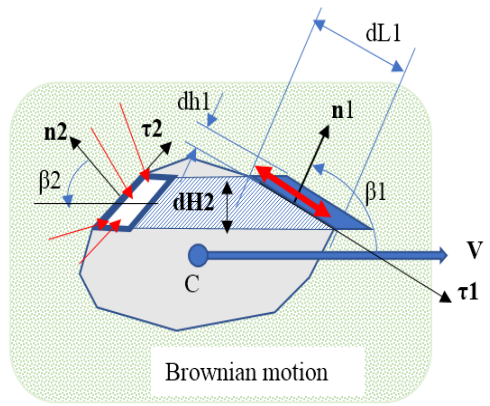

Fig. 2. Interaction of the rectilinear translation (non-rotating) motion body in windless air.

Considering the very small air element in the pressure zone, we use the momentum change in the differential form. Accord- action phenomenon.

The mathematical model is described in the case of definite geometry shaped prism (Figs. 1-3).

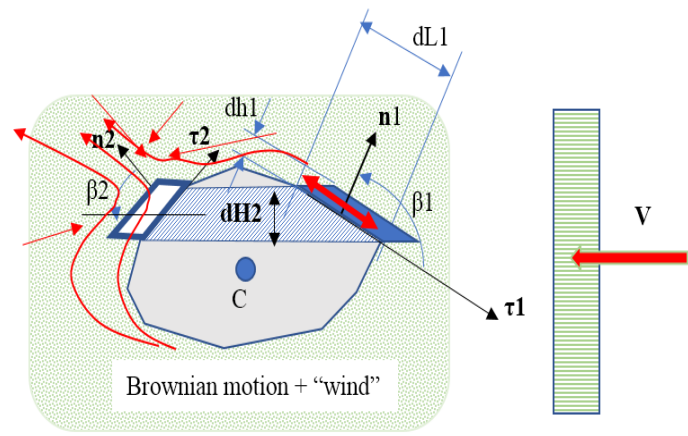

Fig. 3. Model of air interactions with stationary prism.

ing to the principle proposed in the present paper (superposition principle), taking in the projection of the area normal $n 1$, before 
and after collisions (fluid-body interaction), we obtain (1): from Brownian interaction to prism:

$m 10 \cdot V B 1-(-m 10 \cdot V B 1)=-N 1 \cdot d t$,

$m 10=V B 1 \cdot d t \cdot d L 1 \cdot B \cdot \rho$,

$p 10=\frac{|N 1|}{d L 1 \cdot B}$,

where $m 10$ - Brownian interaction mass; $V B 1$ - an average air normal velocity in the pressure zone; $N 1$ - normal force to a small element from the air; $d t$ - an infinitive small time interval; $d L 1$ - width of a small element; $B$ - prism height perpendicular to the plane of motion; $\rho$ - density of air; $p 10$ - atmospheric pressure in the pressure zone.

From prism air interaction at windward side (pressure side) (2):

$$
\begin{aligned}
& m 1 \cdot v \cdot \cos (\beta 1)-0=-\Delta N 1 \cdot d t, \\
& m 1=v \cdot \cos (\beta 1) \cdot d t \cdot d L 1 \cdot B \cdot \rho, \\
& \Delta p 1=\frac{|\Delta N 1|}{d L 1 \cdot B},
\end{aligned}
$$

where $m 1$ - prism and air interaction boundary layer mass; $v$ - prism velocity; $\beta 1-$ an angle between velocity $v$ and normal $n 1$; $\Delta N 1$ - additional normal force acting on a prism; $\Delta p 1$ - pressure increase in the pressure zone.

From the system of six equations (1), (2) it is possible to find six unknowns. The two unknowns are required to solve the following calculations:

$p 10=2 \cdot V B 1^{2} \cdot \rho \cdot d t$,

$\Delta p 1=\rho \cdot d t \cdot v^{2} \cdot[\cos (\beta 1)]^{2}$.

Accordingly, it is possible to apply equations like (1)-(4) in the suction zone (leeward side). However, the task is little complicated with the number of momentum differentials in the suction zone: it is not clear how to simplify the equations in differential form. Therefore, it is suggested to use one or the other hypothesis. Hypotheses should be tested experimentally or by using computer applications.

$p 20=2 \cdot V B 2^{2} \cdot \rho \cdot d t ;$

In the suction zone, pressure reduction $\Delta p 21$ over the entire surface is constant and proportional to the square of the velocity $v$. We get:

$\Delta p 21=-\rho \cdot C 1 \cdot v^{2}$,

\section{The second hypothesis}

In the suction zone, pressure reduction $\Delta p 22$ over the entire surface is not constant but is proportional to the square of the velocity $v$ and also depends on the normal $n 2$ to the surface area and position angle $\beta 2$. Thus, we obtain (7), (8): where $C 1-$ a constant found according to the experimental or numerical modelling; $V B 2$ - an average air normal velocity in the suction zone.

$$
\begin{aligned}
& \Delta p 22=-\rho \cdot C 2 \cdot v^{2} \cdot \cos (\beta 2), \\
& p 20=2 \cdot V B 2^{2} \cdot \rho \cdot d t,
\end{aligned}
$$

where $C 2$ is the second constant found in the same way as $C 1$. 
The obtained differential relationships (3)-(8) can be used in the engineering analysis and synthesis tasks in the low speed range. The prism parameters of the given configuration must be used along with the solution for the integral equations for the object surface.
For practical engineering calculations, it is recommended to adopt $V B 1=V B 2$ for low velocity $v<<V B 1$ and $v<<V B 2$ ranges. Then $p 01=p 02=p 0$, where $p 0$ is the mean atmospheric pressure around the given prism.

\section{STATIONARY BODY (PRISM) IN AIRFLOW}

Looking into the interaction phenomenon of airflow with a fixed prism (Fig. 3.), it appears that the interaction process might be different, as discussed above for a rectilinear translation motion. This is because air particles have extra velocity and extra kinetic energy. However, taking the concept of interactions in relative motion, the formulas obtained previously (3)-(8) can then be used in the engineering calculations, were is air flow velocity.

\section{MOVING BODY (PRISM) IN AIRFLOW}

Two cases of prism motion discussed above (i.e., a rectilinear translation motion of prism in still air and a fixed prism in air- flow) allow for the formulation of the interaction of a moving prism in a moving air stream (Fig. 4).

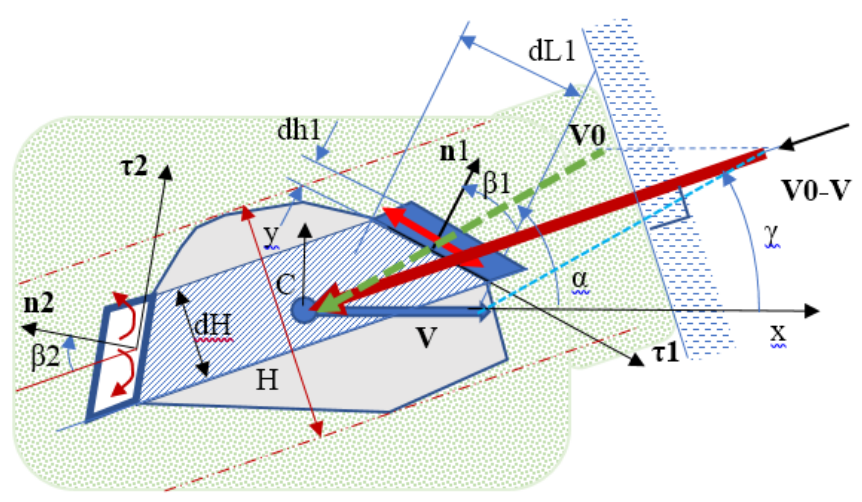

Fig. 4. Model of a moving prism in the airflow.

For this purpose, the relative motion velocity $V r$ vector in the pressure zone must be recalculated by determining the angle $\gamma$ from the elementary parallelograms with normal directions $n 1$ and $n 2$ (Fig. 4). By plotting the vectors $V$ and $V 0$ on the $x$ and $y$ axes, we obtain (9), (10): 


$$
\begin{aligned}
& V r=\sqrt{(-V 0 \cdot \cos (\alpha)-V)^{2}+(-V 0 \cdot \sin (\alpha))^{2}}, \\
& \cos (\gamma)=\frac{-V 0 \cdot \cos (\alpha)-V}{\sqrt{(-V 0 \cdot \cos (\alpha)-V)^{2}+(-V 0 \cdot \sin (\alpha))^{2}}},
\end{aligned}
$$

where $V r$ - a relative velocity module; $V 0$ - wind velocity as a vector; $V$ - prism rectilinear translation motion velocity as a vector and $\alpha$ is a $V 0$ flow angle (Fig. 4).

By using the obtained relationships (3)-(8), it is possible to solve various technical tasks of fluid (air) and prism motion for engineers. For example, it is possible to carry out motion analysis tasks where airflow resistance forces can be obtained. It is even more important for solving the prob- lems of energy extraction from a fluid (air) stream. Additionally, it is also possible to optimize the shape of the object (prism) in order to obtain the desired effect (criterion) along with motion control task optimization and real system synthesis by using mechatronic systems.

\section{NUMERICAL EXPERIMENT}

In the proposed airflow interaction averaging superposition method, the engineer calculations require the selection of an approximation model, which in turn requires numerical values of constants $C 1$ or $C 2$.

Thereby, in this direction 3D-RANS equation was solved by using K- $\varepsilon$ realiz- able turbulence model. The sharp prism with two sides (other than the longest side) was $0.16 \mathrm{~m}$ and the depth was maintained constant at $0.16 \mathrm{~m}$. In the given numerical experiment, knowing the total flow force $F x$, constant $C 1$ was found according to the formulas above. The numerical values of $F x$ and constant $C 1$ are given in Figs. 5, 6.

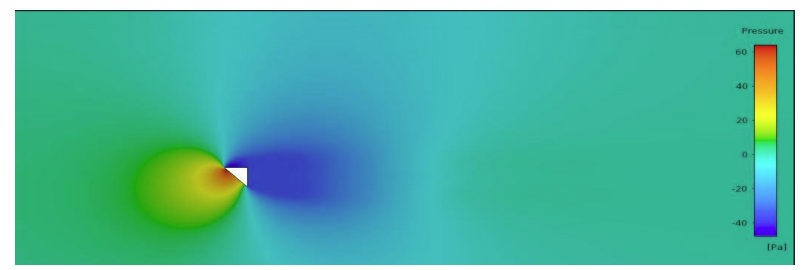

Fig. 5. Sharp right-angle Isosceles prism (angles $45^{\circ}, 45^{\circ}, 90^{\circ}$ ) with leading vertex $45^{\circ}$ in stationary airflow. Quality distribution of pressure in suction and compression zones. $F x 1=1.8942 \mathrm{~N} ; C 1=0.238$.

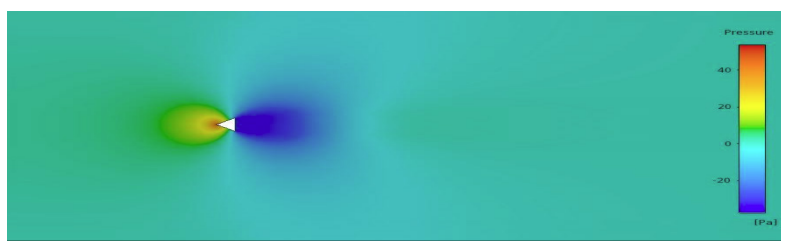

Fig. 6. Equilateral triangle (angles $60^{\circ}, 60^{\circ}, 60^{\circ}$ ) sharp prism in airflow with leading vertex $60^{\circ}$. Distribution of pressure in suction and compression zones. $F \times 2=1.3680 \mathrm{~N} ; C 1=0.245$. 
1. Theoretical calculations for the $2 \mathrm{D}$ model differ from the 3D numerical simulation.

2. Visually, the first and second hypotheses $(\mathrm{C} 1, \mathrm{C} 2)$ come true because the pressure in the suction zone is approximately the same at all locations (Figs. 4, 5).

3. Pressure zone in different local places is different.

4. In spite of the deviations of the pressure from the constant along the edges of the pressure zone of the prism, the total force can be found according to the methodology proposed here, for a constant narrow range: $0.238<C 1<0.245$ (Figs. 5, 6).

5. It is known from scientific experimental studies that the difference between 3D and 2D drag coefficients is about twice. Therefore, $C \approx 0.5$ should be used in approximately $2 \mathrm{D}$ calculations; this value is further used in all future calculations.

\section{OPTIMIZATION OF TRIANGLE PRISM IN RECTILINEAR TRANSLATION MOTION IN AIRFLOW}

The motion model with given length and angles is shown in Fig. 7, where $R^{(e)}$ and $M_{Z}{ }^{(e)}$ are the external forces and moment, providing a rectilinear translation motion of a triangle prism.

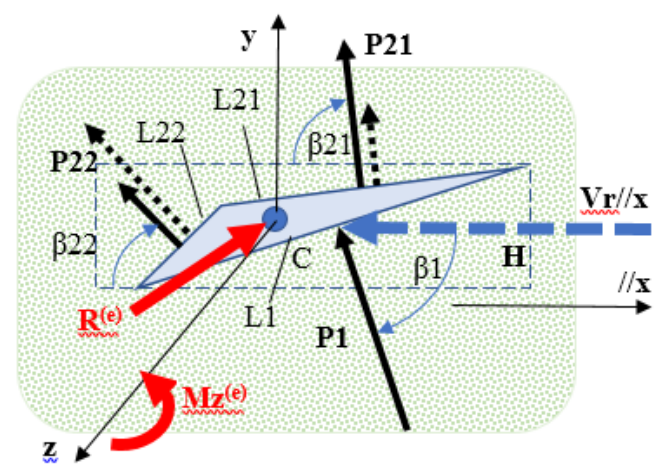

Fig. 7. Triangle prism movement in the wind direction $\mathrm{Vr} / / \mathrm{V} / / \mathrm{x}$.

The optimization task should determine the prism braking velocity $V$ that provides the maximum power in the mechatronic system. The differential equation of motion will be (11):

$m \cdot \ddot{x}=-(V 0-V)^{2} \cdot C 0+Q$.
Here, $C 0=B \cdot \rho \cdot\left[(\cos (\beta 1))^{3} \cdot L 1+C 2\right.$.
$\left.\cdot(\cos (\beta 21))^{2} \cdot L 21+C 2 \cdot(\cos (\beta 22))^{2}\right] \cdot L 22$

where $\mathrm{m}$ is a prism mass; $\ddot{x}$ - constrained acceleration along x axis; $Q$ - braking force. 
The task of optimization is the following.

Given for motion parameters $C 0, m$ for stationary motion, when acceleration is zero $(\ddot{x}=0)$, the force $Q$ of the prismatic

$V=\frac{V 0}{3}, \quad P=\frac{4 \cdot C 0 \cdot V 0^{3}}{27}, \quad Q=\frac{4 \cdot C 0 \cdot V 0^{3}}{9}$.

As can be inferred from Eq. (12), (if the flow rate $V 0$ changes) the real system braking mechatronic system shall be found to provide the maximum power $P$ produced by the airflow force.

In the given case, without limitation, there is an optimal solution as given by (12):

requires a mechatronic force control $Q$ system.

\section{MOTION OF A SHARP PRISM IN A VERTICAL PLANE}

We observe the movement of a sharp prism in a vertical plane, when angles are equal $\beta 22=\beta 21=\beta 1$ (Fig. 8).

According to the theory of the first model mentioned above (use of a constant $C 1$ ), the motion of a sharp prism in a vertical plane is described by differential equations (13), (14):

$$
\begin{aligned}
m \cdot \ddot{x}= & -\left[\rho \cdot L \cdot B \cdot(\dot{x} \cdot \sin (\alpha)-\dot{y} \cdot \cos (\alpha))^{2} \cdot(1+C 1) \cdot \sin (\alpha)\right. \\
& \cdot \operatorname{sign}(\dot{x} \cdot \sin (\alpha)-\dot{y} \cdot \cos (\alpha)) \cdot \sin (\alpha)], \\
m \cdot \ddot{y}= & {\left[\rho \cdot L \cdot B \cdot(\dot{x} \cdot \sin (\alpha)-\dot{y} \cdot \cos (\alpha))^{2} \cdot(1+C 1) \cdot \sin (\alpha)\right.} \\
& \cdot \operatorname{sign}(\dot{x} \cdot \sin (\alpha)-\dot{y} \cdot \cos (\alpha)) \cdot \cos (\alpha)]-m \cdot g,
\end{aligned}
$$

where $\ddot{x}$ and $\ddot{y}$ are acceleration projections; $\alpha$ - an angle between normal and vertical direction; sign is a \pm 1 depending on the function in brackets; $g$ - free fall acceleration.

The obtained equations allow solving analytical and parametric optimization problems for a given non-stationary motion case. In addition, it should be noted that this movement is close to the bird's gliding or

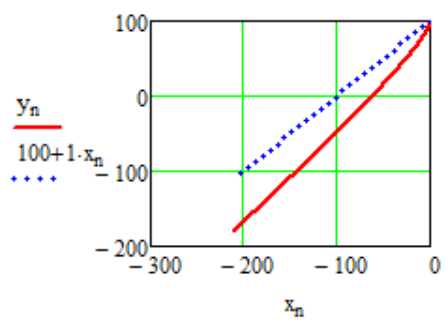

Fig. 8. The trajectory of the centre of mass motion in the vertical plane, starting from the coordinates $(x, y)=(0,+100)$. diving movement in the air.

Example of diving motion calculation is shown below (Figs. 8-11). All parameters in system SI: $\rho=1.25 ; L \cdot B=0.04 ; m \cdot g=2 ; C 1=0.5 ; \alpha=\frac{\pi}{4}$.

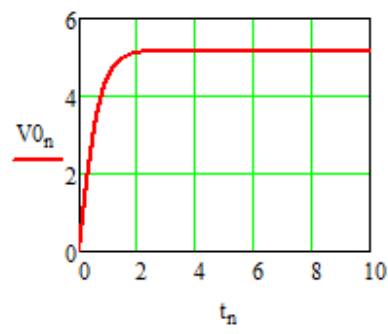

Fig. 9. Speed projection on prism normal. 


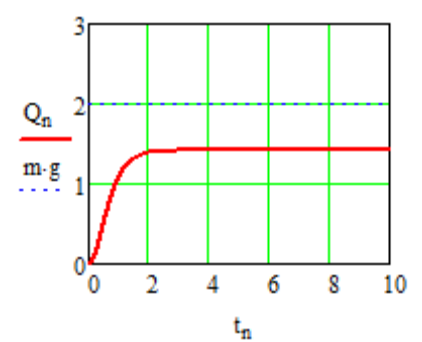

Fig. 10. Frontal force of air interaction.

The obtained modelling results show that the velocity projection perpendicular to the sharp prism reaches the terminal value and does not change further. However, there is no acceleration component in this direction. Accordingly, the acceleration in the

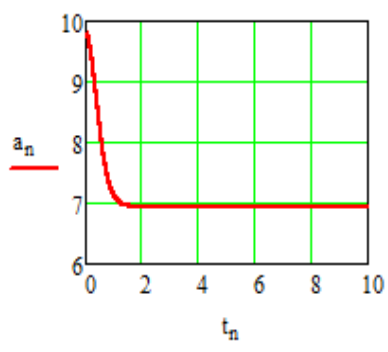

Fig. 11. Total acceleration of prism fall and lateral slip. The figure shows that the viscous forces mentioned at the beginning of the article are not taken into account.

tangent direction becomes constant, but the velocity component increases linearly with time. This means that in real-world applications, viscosity must be observed or a real prism with a blunt front surface must be considered.

\section{SYNTHESIS AND OPTIMIZATION TASK OF ENERGY EXTRACTION}

For the description of the given problem, we will use the previously obtained relation for sharp triangular prism 1 , whose motion is relative to wind flow 2 (Fig. 12). The movement is along the $\mathrm{x}$ axis an angle $\alpha$ to the flow. We consider the case where the prism is attached to a base with elastic spring 3 . The energy is obtained by electrodynamic braking system 4. According to expressions such as (11), the differential equation of motion is (15):

$$
\begin{aligned}
m \cdot \ddot{x}= & -f(x)-D(\dot{x})-A \cdot \rho \cdot(V 0 \cdot \cos (\beta-\alpha)+ \\
& +\dot{x} \cdot \cos (\beta))^{2} \cdot \operatorname{sign}\left(V 0 \cdot \cos (\beta-\alpha)+\dot{x} \cdot \cos (\beta)^{2},\right.
\end{aligned}
$$

where $f(x)$ - a spring characteristic; $D(\dot{x})$ - force of an electrodynamic braking system; $A$ - a prism area of the pressure zone, as before: $A=L \cdot B$.

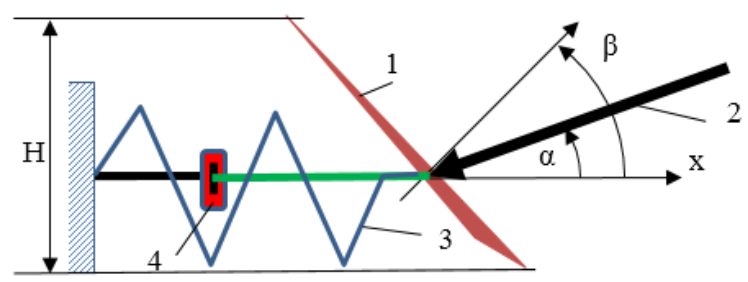

Fig. 12. Model showing the working principle for energy extraction using the phenomenon of air-body (prism) interaction with sharp triangular prism 1, wind flow 2, elastic spring 3, and electrodynamic braking system 4. 
The resulting equation (15) allows solving all kinds of analytical, optimization and synthesis problems if a criterion with given constraints on system and control parameters is formulated.

As an example, we solve a synthesis problem where there can be change in area A realized by using the perforated plate. Taking into account case of linear spring with a linear electrodynamic braking system, equation (15) is expressed as (16):

$$
\begin{aligned}
m \cdot \ddot{x}= & -c \cdot x-b(\dot{x}) \cdot \dot{x}-A(\dot{x}, x, t) \cdot \rho(V 0 \cdot \cos (\beta-\alpha)+\dot{x} \cdot \cos (\beta))^{2} \\
& \cdot \operatorname{sign}\left(V 0 \cdot \cos (\beta-\alpha)+\dot{x} \cdot \cos (\beta)^{2},\right.
\end{aligned}
$$

where $c$ - elasticity of spring; $b(\dot{x})$ - electrodynamic system control force; $A(\dot{x}, x, t)$ - area variation control action depending on the synthesis task of velocity, coordinate and time.

The energy optimization task should analyse the power $P$ of the electrodynamic system (17):

$$
P=d(\dot{x}) \cdot \dot{x}^{2}
$$

\section{MODELLING OF VARIABLE AREA PRISM IN AIRFLOW}

Let us consider the possibility of obtaining energy from the flow if the change of area occurs instantaneously when opening or closing the perforation. Let us assume the simplest direction of the wind flow parallel to the prism motion axis $\alpha=0$ and with optimal angle $\beta=0$ (Fig. 12). Then the differential equation of motion will be (18):

$$
\begin{aligned}
m \cdot \ddot{x}= & -c \cdot x-b \cdot \dot{x}-(1+C 1) \cdot A \cdot(a 0-a 1 \cdot \operatorname{sign}(\dot{x}+\Delta v)) \\
& \cdot \rho(V 0+\dot{x})^{2} \cdot \operatorname{sign}(-V 0-\dot{x})
\end{aligned}
$$

where $b, C 1, a 0, a 1, \Delta v$ are constants.

Equation (18) has to be solved numerically by estimating the efficiency of energy production by criterion (17) as power.

Modelling example for a small plate is given below; all values are used in SI system. The results obtained are shown in Figs. 13-16 for parameters: $m=0.5 \mathrm{~kg}$; $c=80 \mathrm{~kg} \cdot \mathrm{sec}^{-2} ; b=0.5 \mathrm{~kg} \cdot \mathrm{sec}^{-1} ; C 1=0.5$; $A=0.04 \mathrm{~m}^{2} ; a 0=0.75 ; a 1=0.25 ; \Delta v=0.5 \mathrm{~m}$ $\mathrm{sec}^{-1} ; \rho=1.25 \mathrm{~kg} \mathrm{~m}^{-3} ; V 0=10 \mathrm{~m} \mathrm{sec}^{-1}$. 


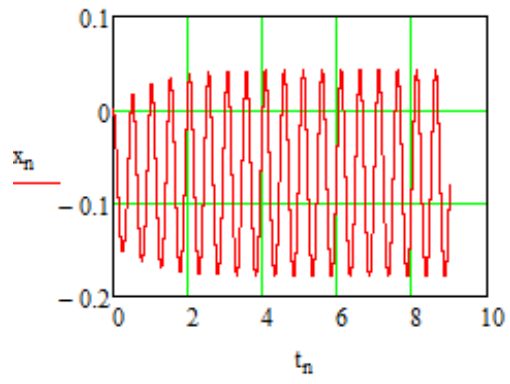

Fig. 13. Displacement with respect to time for a variable area prism.

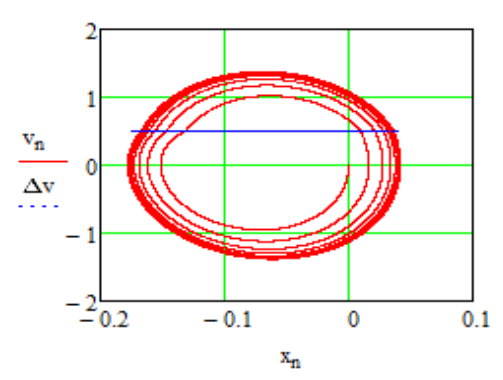

Fig. 15. Phase plane motion.

From the graphs in Figs. 13-16, the following conclusions can be drawn:

1. In the aerodynamic system it is possible to initiate a stable oscillation movement by changing the interaction area of the perforated plate.

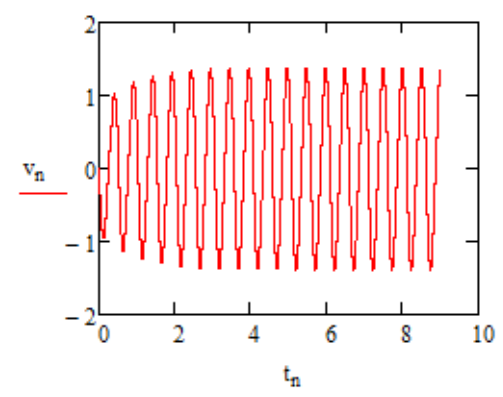

Fig. 14. Velocity with respect to time for a variable area prism.

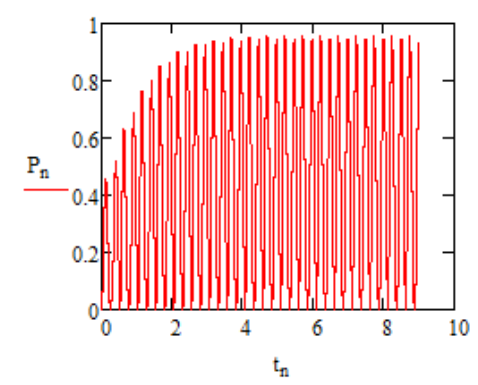

Fig. 16. Power generated for the given time interval.

2. The opening and closing of perforations may be affected by a mechatronic system.

3. There is the second mechatronic system for generating energy, which breaks the speed of oscillation and generates power.

\section{RESULTS AND DISCUSSION}

Within the framework of the present study, we have developed a new method for calculating the approximate flow and rigid body interaction for: a moving body in the low speed air, a stationary body in the airflow and moving prism in the airflow. The fluid flow is assumed to be laminar, incompressible, and viscous nature of the fluid is not taken into account for mathematical modelling, as an alternative the coefficient of interaction (constant) is introduced from numerical simulation results. Stable result in case of a variable area prism for energy extraction has been observed (see Fig. 15). Though the power obtained is small, the proposed theory (superposition principle) helps identify the cause and make a way for overall better efficiency as the nature of the proposed principle is purely mathematical and is explained in an easily understandable 
and straightforward way. Good and satisfactory results are obtained both for moving prisms in the airflow and energy extraction by using a variable area prism. An alternate approach to a solid body-fluid (air) interac- tion phenomenon other than the traditional vortex induced vibration techniques has been found to be successful in calculating energy extraction by means of a simple mathematical model.

\section{CONCLUSIONS}

The method developed in the present study helps perform tasks of analysis, optimization and synthesis for the interaction of objects with fluids in a simplified way, only for rectilinear translation motion of body. The proposed techniques help overcome the extensive space-time programming methods. The analysis, optimization and computer modelling examples discussed in this paper illustrate the effectiveness of the pro- posed method in engineering calculations.

The present study also establishes the fact that there is an alternate approach to vortex induced vibration phenomenon for the energy extraction process considering just the body-fluid interaction coefficient in a straightforward mathematical model. The theory can be extended to any complex body-fluid interaction as the work is purely mathematical in nature.

\section{ACKNOWLEDGEMENTS}

This research has been funded by the Latvian Council of Science, project "Creation of Design of Experiments and Metamodelling Methods for Optimization of Dynamics of Multibody 3D Systems
Interacting with Bulk Solids and Fluids", project No. Lzp-2018/2-0281 and supported by the Doctoral Grant programme of Riga Technical University.

\section{REFERENCES}

1. Wei, G. (2005). A Fixed-Mesh Method for General Moving Objects in Fluid Flow. International Journal of Modern Physics B, 19 (28), 1719-1722.

2. Wick, T. (2011). Fluid-Structure Interactions Using Different Mesh Motion Techniques. Journal of Computers and Structures, 89 (13), 1456-1467.

3. Codina, R., Houzeaux, G., CoppolaOwen, H., \& Baiges, J. (2009). The FixedMesh ALE Approach for the Numerical Approximation of Flows in Moving Domains. Journal of Computational Physics, 228 (5), 1591-1611.
4. Tezduyar, T. E., Sathe, S., Keedy, R., \& Stein, K. (2006). Space-Time Finite Element Techniques for Computation of FluidStructure Interactions. Comput. Methods Appl. Mech. Eng., 195, 2002-2027.

5. Tezduyar, T. E., \& Sathe, S. (2007). Modelling of Fluid-Structure Interaction with the Spacetime Finite Elements: Solution Techniques. Int. J. Numer. Meth. Fluids, 54, 855-900.

6. Han, D., Liu, G. R., \& Abdallah, S. (2020). An Eulerian-Lagrangian-Lagrangian Method for 2D Fluid-Structure Interaction Problem with a Thin Flexible Structure Immersed in Fluids. Computers \& Structures, 228, 106179. 
7. Takizawa, K, \& Tezduyar, T. E. (2011). Multiscale Space-Time Fluid-Structure Interaction Techniques. Comput. Mech., 48, 247-267.

8. Neumuller, M., \& Steinbach, O. (2011). Refinement of Flexible Space-Time Finite Element Meshes and Discontinuous Galerkin Methods. Computing and Visualization in Science, 14, 189-205.

9. Gerstenberger, A., \& Wall, W. A. (2006). An Extended Finite Element Method Based Approach for Large Deformation FluidStructure Interaction. European Conference on Computational Fluid Dynamics. ECCOMAS CFD, Netherlands, TU Delf.

10. Behr, M. (2008). Simplex Space-Time Meshes in Finite Element Simulations. International Journal for Numerical Methods in Fluids, 57 (9), 1421-1434.

11. Danwitz, M. V., Karyofylli, V., Hoster, N., \& Behr, M. (2019). Simplex Space-Time Meshes in Compressible Flow Simulations. International Journal for Numerical Methods in Fluids, 91 (1), 29-48.
12. Diosady, L.T., Murman, S.M., \& Carton de Wiart, C. (2018). A higher order space time finite element method for moving body and fluid structure interaction problem. In 10th International Conference on Computational Fluid Dynamics (ICCFD10) (pp. 1-16), 9-13 July 2018, Barcelona, Spain.

13. Tipans, I., Viba, J., Irbe, M., \& Vutukuru, S. K. (2019). Analysis of Non-Stationary Flow Interaction with Simple Form Objects. Agronomy Research, 17 (1), 1227-1234.

14. Barrero-Gil, A., Pindado, S., \& Avila, S. (2012). Extracting Energy from VortexInduced Vibrations. A Parametric Study. Applied Mathematical Modelling, 36 (7), 3153-3160.

15. Bearman, P.W. (1984). Vortex Shedding from Oscillating Bluff Bodies. Fluid Mech., 16, 195-222.

16. Chin, D. D., \& Lentink, D. (2016). Flapping Wing Aerodynamics: From Insects to Vertebrates. J. Exp. Biol., 219, 920-932. 